\title{
Review of SIS Experimental Results on Strangeness
}

\author{
Helmut Oeschler \\ Institut für Kernphysik, Technische Universität Darmstadt, \\ D-64289 Darmstadt, Germany
}

\begin{abstract}
A review of meson emission in heavy ion collisions at incident energies around $1-2 A \cdot \mathrm{GeV}$ is presented. It is shown how the shape of the spectra and the various particle yields vary with system size, with centrality and with incident energy. A statistical model assuming thermal and chemical equilibrium and exact strangeness conservation (i.e. strangeness conservation per collision) explains most of the observed features.

Emphasis is put onto the study of $K^{+}$and $K^{-}$emission. In the framework of this statistical model it is shown that the experimentally observed equality of $K^{+}$and $K^{-}$rates at threshold corrected energies $\sqrt{s}-\sqrt{s_{t h}}$ is due to a crossing of two excitation functions. Furthermore, the independence of the $K^{+}$to $K^{-}$ ratio on the number of participating nucleons observed between 1 and $10 A \cdot \mathrm{GeV}$ is consistent with this model. The observed flow effects are beyond the scope of this model.
\end{abstract}

\section{Introduction}

Central heavy ion collisions at relativistic incident energies represent an ideal tool to study nuclear matter at high densities and at high temperatures. Particle production is at all incident energies a key quantity to extract information on the properties of nuclear matter under theses extreme conditions. Particles carrying strangeness have turned out to be very valuable messengers. At incident energies around $1-2 A \cdot \mathrm{GeV}$, as available at the SIS accellerator at GSI, Darmstadt, these particles are produced below their threshold in $N N$ collisions. Due to strangeness conservation, a $K^{+}$meson is produced with an associated $\Lambda$, having a threshold of $671 \mathrm{MeV}$ in the centre-ofmass system and $1.58 \mathrm{GeV}$ in the laboratory system. $K^{-}$is "cheapestly" produced by $K^{-} K^{+}$pair creation, yielding thresholds of $987 \mathrm{MeV}$ (c.m. system) and $2.5 \mathrm{GeV}$ (lab system). The subthreshold strangeness production is the key parameter governing this paper. Furthermore, $K^{-}$and $K^{+}$behave very differently in nuclear matter. While $K^{+}$hardly finds a partner to react with, $K^{-}$exhibits a strong interaction with nuclear matter.

In this paper, an overview of the shape of the measured meson spectra is given and their yields are studied as a function of system size $A+A$, of the number of participating nucleons $A_{\text {part }}$ and as a function of incident energy. These results are discussed along with the question whether the observations are in agreement with the assumption of a thermal and chemical equilibrium. Of special interest is the ratio of $K^{+} / K^{-}$yields as the measured values in heavy ion collisions differ strongly from those in pp reactions. These findings have lead to the interpretation that in heavy ion collisions the "effective masses" of $K^{+}$and $K^{-}$might be changed as predicted 
for dense nuclear matter. It turns out that a statistical approach is very successful to explain the measured yields.

\section{Experimental Devices}

At GSI, Darmstadt, three major experimental devices for measuring strange particle production are installed:

- The FOPI setup, a $4 \pi$ detector, designed to study the global features of heavy ion collisions at these energies [1]. This detector measures all particles and is optimized for flow studies and informations related with the reaction plane. It is also suited for vertex reconstruction to detect $K^{0}, \Lambda$, etc. In the present setup kaons can only be detected at backward rapidities. A major upgrade is under way to improve the detection and trigger capabilities.

- The magnetic Kaon Spectrometer KaoS specially designed to study rare kaon production [2]. It is optimized for short flight paths because of the kaon decay. It has a very selective trigger to handle high beam intensities. Multiwire chambers for tracking are installed to reduce the background in kaon measurements with $K^{+}$rates $10^{5}$ times smaller than for protons. Two detector arrays serve for centrality selection and reaction-plane determination. Most measurements are performed around midrapidity; in some cases a large fraction of the full phase space has been studied by moving the spectrometer.

- The TAPS detector consists of six arrays of $\mathrm{BaF}_{2}$ modules measuring $\gamma$ rays. It detects among others the $\eta$ which decays by $\gamma$ emission. $\eta$ has net strangeness zero, but contains $s \bar{s}$ pairs.

Since this experimental review is restricted to spectral shapes and yields, mainly results from the KaoS collaboration are shown. Very interesting flow studies are given in the two following talks by P. Crochet and by Y. Shin.

\section{Spectra and Yields}

Spectra of $K^{+}$from mass symmetric systems $\mathrm{C}+\mathrm{C}$ and $\mathrm{Au}+\mathrm{Au}$ at incident energies from 0.6 to $2.0 A \cdot \mathrm{GeV}$ measured at midrapidity are shown in Fig. 1. exhibiting Boltzmann shapes [3, 何. Their inverse slope parameters increase monotoneously with incident energy and the heavier system exhibits harder spectra than the light system at the same incident energy.

Pion spectra show deviations from a Boltzmann shape [5, 6, 7]. Their shapes are qualitatively understood as composed of two parts, a direct (or thermal) pion contribution and pions from decaying baryonic resonances, which produce an enhancement at lower kinetic energies 8, 9, 10. The slopes of the high-energy component agree well with those extracted from the corresponding $K^{+}$spectra.

Next, we study the inclusive yields. In many cases the phase-space distribution has been measured, yielding slight anisotropies. These effects are taken into account to obtain the inclusive cross sections and are in the order of at most $30 \%$ as compared to a mid-rapidity measurement extrapolated to $4 \pi$ assuming isotropic emission [3, 何. These cross sections are divided by the geometrical reaction cross sections (using $r_{0}=1.2 \mathrm{fm}$ ) to obtain multiplicities. For a comparison of different systems these multiplicity values are divided by the system mass $A$ as shown in Fig. 2 2 for 1 and 


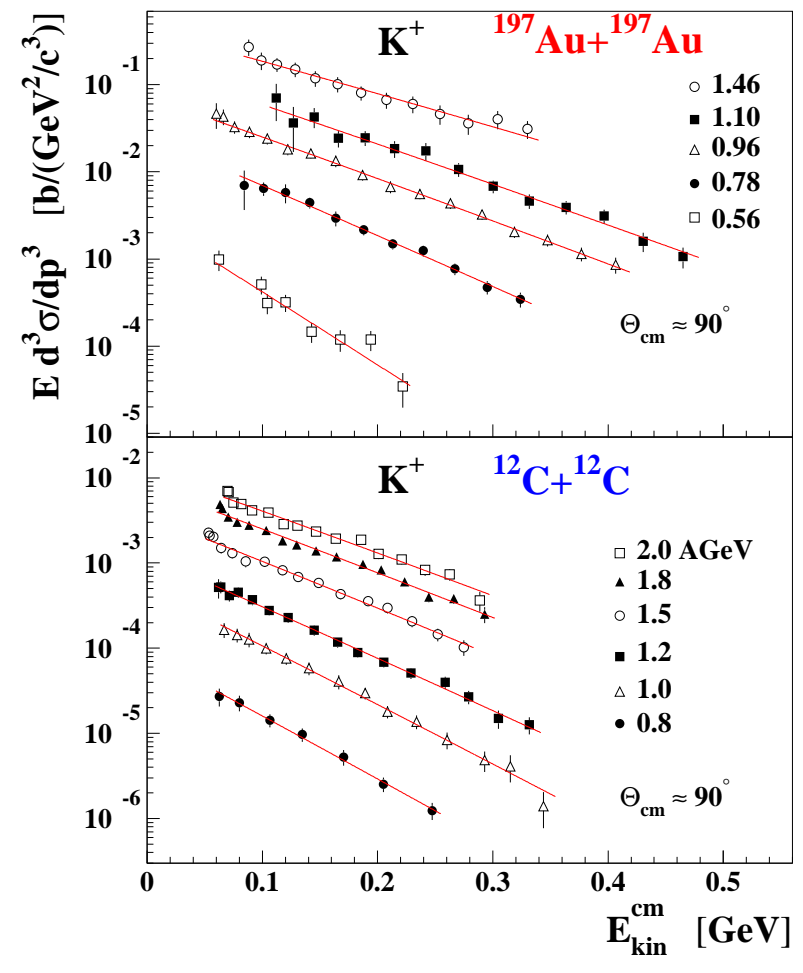

Figure 1. $K^{+}$spectra measured at midrapidity for $\mathrm{Au}+\mathrm{Au}$ (upper part) and $\mathrm{C}+\mathrm{C}$ (lower part) at various incident energies 3 , 1. The solid lines are Boltzmann fits to the data.

1.5 $A \cdot \mathrm{GeV}$. A striking difference is seen between the trends observed for $K^{+}$and $K^{-}$which exhibit a strong rise of $M / A$ with system size and pions which show a slight decrease. The values observed for $\eta$ production seem to be rather constant. Some $\eta$ measurements were not fully inclusive. Those values have been corrected. Having in mind that kaons are produced below their $N N$ thresholds, one can think of mechanisms causing such a rise: Only multiple collisions can collect the energy needed for kaon production and the probability for multistep interactions increases with density and/or system size.

It is of interest to follow how the mass dependence varies with incident energy. The upper part of Fig. 3 shows the pion and $K^{+}$multiplicities per $A$ for $\mathrm{C}+\mathrm{C}$ and $\mathrm{Au}+\mathrm{Au}$ collisions as a function of incident energy. The pion multiplicities $\left(M_{\pi} / A\right)$ representing the sum of charged and neutral pions 洵, are smaller for $\mathrm{Au}+\mathrm{Au}$ than for $\mathrm{C}+\mathrm{C}$ whereas the $K^{+}$multiplicities per $A$ exhibit the opposite behaviour. These trends are already seen in Fig. 2. With increasing incident energy the differences in $M / A$ between the light and the heavy system become smaller as can be seen in Fig. 3 .

The mass dependence of the $K^{+}$yields is demonstrated in the lower part of Fig. 3 showing the ratio $(M / A)_{\mathrm{Au}+\mathrm{Au}} /(M / A)_{\mathrm{C}+\mathrm{C}}$. The enhancement factor for $K^{+}$reaches 6 at the lowest measured incident energy. At lower incident energies more and more individual $N N$ interactions are needed to accumulate the energy to produce a $K^{+}$ and hence a increasing sensitivity to density occurs. These data represent therefore the ideal set of information to extract the nuclear equation of state (EOS). Depending on the stiffness of the EOS different densities might be reached. A soft EOS allows to compress the nuclear matter easier, higher densities can be achieved and as a 

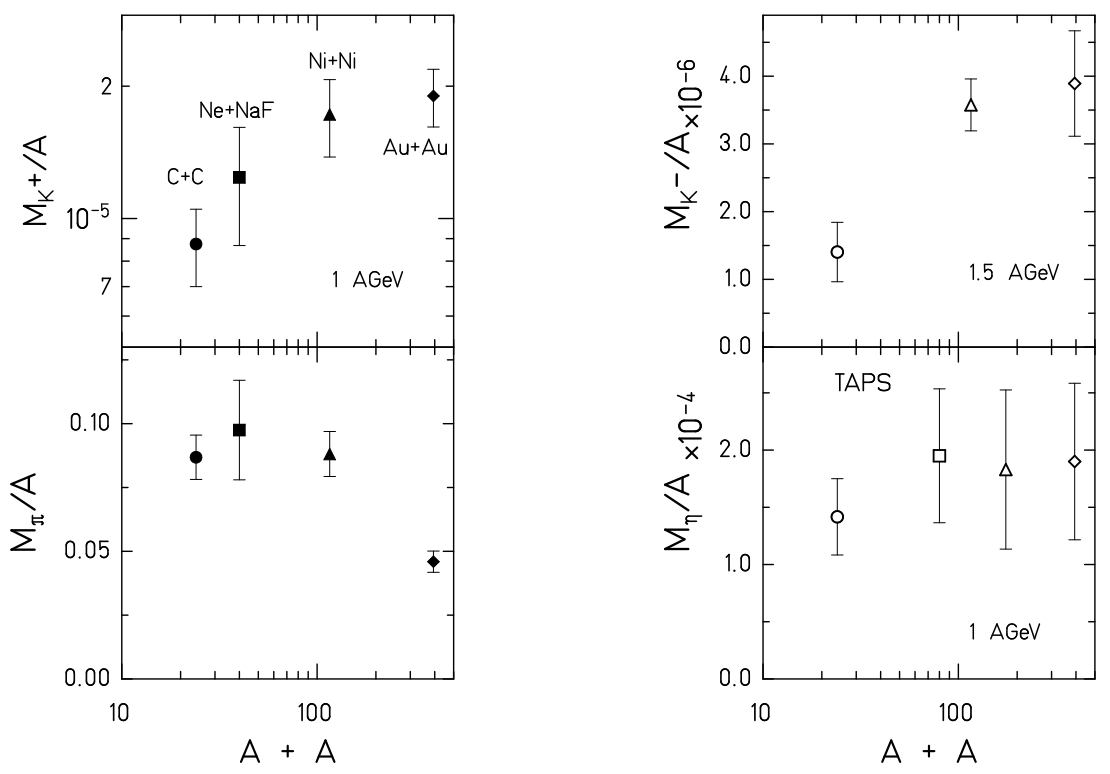

Figure 2. Multiplicities per mass number $A$ as a function of $A+A$ for $K^{+}$and pions $\left(\pi^{+}, \pi^{0}, \pi^{-}\right)$(at $1 A \cdot \mathrm{GeV}$ ) on the r.h.s and $K^{-}$(at $1.5 A \cdot \mathrm{GeV}$ ) and $\eta$ (at 1 $A \cdot \mathrm{GeV})$ left. Partially preliminary data from $3,1,6,13,15,16$. . Particles with strangeness exhibits a strong rise while those with $\mathrm{s}=0$ do not.

consequence more $K^{+}$can be produced. Yet, qualitative arguments are not sufficient to extract the stiffness parameter; transport model calculations are needed and they show that these data are in agreement with a soft $(k=200 \mathrm{MeV})$ EOS [11. This conclusion, however, is put in question by a recent study 12 .

Negatively charged kaons are at SIS energies always produced below their $N N$ threshold and rather small cross sections are expected in heavy ion collisions. Furthermore, $K^{-}$can be easily absorbed in nuclear matter. Yet, the measured yields turned out to be rather high compared to the yields measured in pp collisions. These measurements by the KaoS Collaboration have attracted considerable interest as in heavy ion collisions the $K^{-}$yield compared to the $K^{+}$cross section is much higher than expected from $N N$ collisions [3, 13. This is especially evident, if the kaon multiplicities are plotted as a function of $\sqrt{s}-\sqrt{s_{t h}}$ where $\sqrt{s_{t h}}$ is the energy needed to produced the respective particle in $N N$ collisions taking into account the mass of the associately produced partner. The obvious contrast between $N N$ and $A A$ collisions, shown in Fig. A, has lead to the interpretation of the results by in-medium properties which cause e.g. a lower threshold for $K^{-}$production when produced in dense matter [17. Of course, the difference between $N N$ and $A A$ collisions as shown in Fig. 团, is not sufficient to conclude on properties of kaons in matter. In heavy ion collisions, kaons can be produced by other channels, e.g. $\pi \Lambda \rightarrow K^{-} N$ which are not available in $N N$ collisions. Only by using detailed transport model calculations one can conclude on new properties of kaons in matter [17]. They demonstrate that by including secondary collisions and the newly opened channels the measured $K^{-}$yields are not reproduced when using bare kaon masses. 

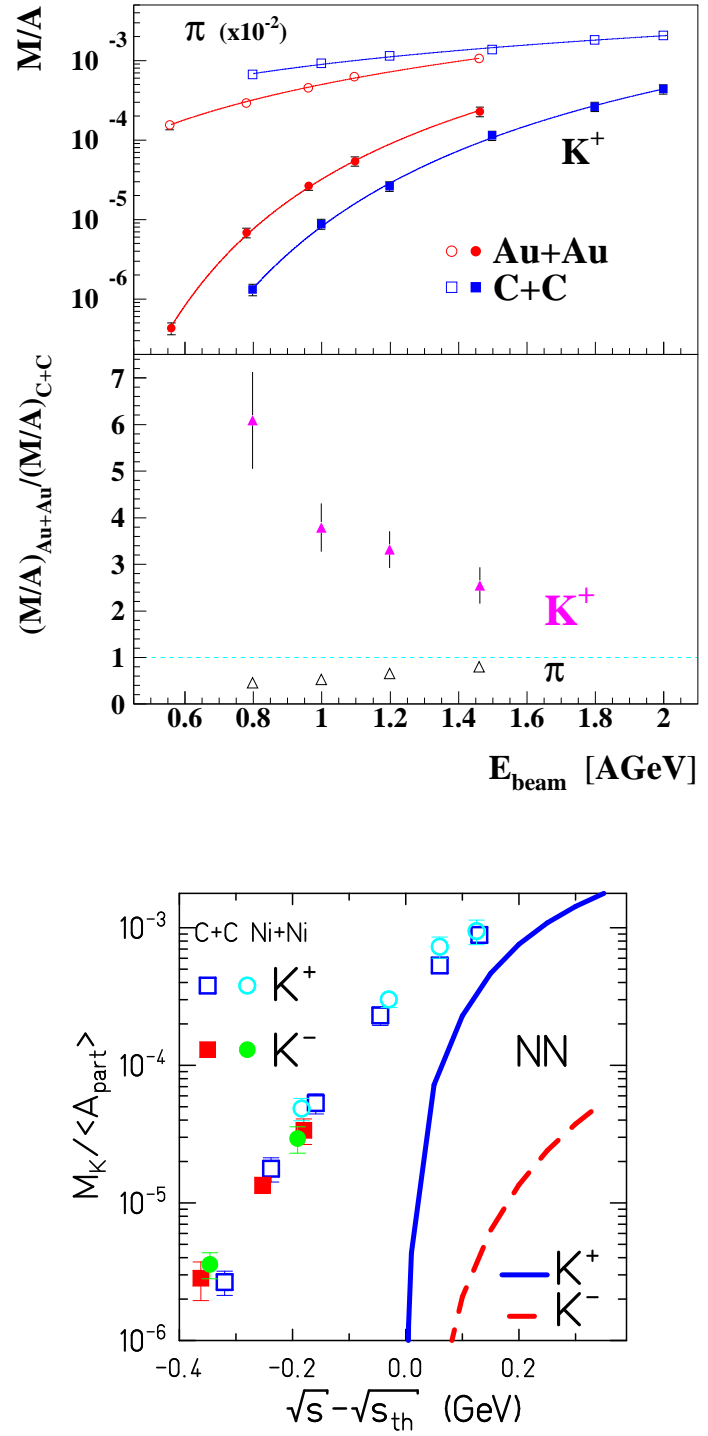

Figure 3. Upper part: Multiplicities of $K^{+}$and pions per $A$ as a function of incident energy. The pion data include charged and neutral pions. The lines represent fits to the data (see 4). Lower part: Ratio of the multiplicities per $A$ $(\mathrm{Au}+\mathrm{Au}$ over $\mathrm{C}+\mathrm{C})$ as a function of incident energy.

Figure 4. Measured $K^{+}$and $K^{-}$yields in heavy ion and $N N$ collisions as a function of $\sqrt{s}-$ $\sqrt{s_{t h}}$ [3, 13, 14. < $\quad A_{\text {part }}>$ is $A / 2$ for heavy ion data and 2 for $N N$ collisions.

\section{Interpretation within a Statistical Model}

The spectral shapes of particles presented so far, are in agreement with the interpretation within a statistical concept. The measured multiplicities for pions and $K^{+}$(Fig. 2) evidence a strong contrast; while the pion multiplicity decreases with the mass of the colliding system, the $K^{+}$multiplicity rises strongly. The latter observation seems to be in conflict with a thermal interpretation, which - in a naive view - should give multiplicities per mass number $A$ being constant.

Usually, the particle number densities or the multiplicities per $A_{\text {part }}$, here for pions, are described in a simplified way by a Boltzmann factor

$$
\frac{M_{\pi}}{A_{\text {part }}} \sim \exp \left(-\frac{<E_{\pi}>}{T}\right) .
$$




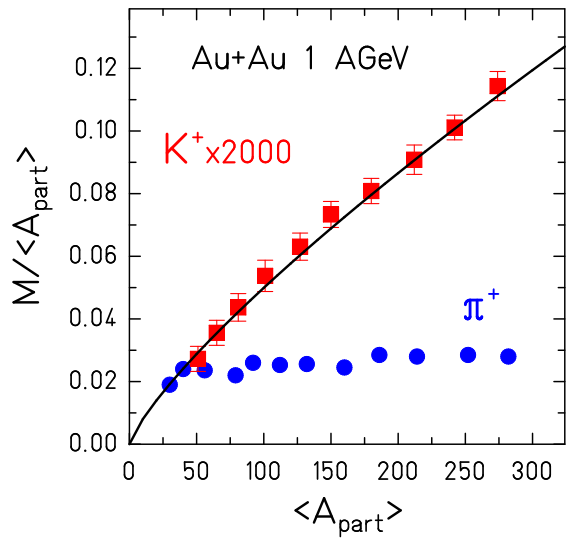

Figure 5. The multiplicity of $K^{+} / A_{\text {part }}$ rises strongly with $A_{\text {part }}$ in contrast to the pion multiplicity 23. This rise can be descibed by the thermal model including exact strangeness conservation (see text).

The production of strange particles has to fulfil strangeness conservation. The attempt to describe the measured particle ratios including strange hadrons at AGS and SPS using a strangeness chemical potential $\mu_{S}$ is quite successful [18, 19]. However, this grand-canonical treatement is not sufficient, if the number of produced strange particles is small. There, a statistical model has to take care of exact strangeness conservation in each reaction as introduced by Hagedorn 20]. This is done by taking into account that together with each $K^{+}$another strange particle, e.g. a $\Lambda$ is produced:

$$
\frac{M_{K^{+}}}{A_{\text {part }}} \sim \exp \left(-\frac{<E_{K^{+}}>}{T}\right)\left[g_{\Lambda} V \int \frac{d^{3} p}{(2 \pi)^{3}} \exp \left(-\frac{\left(E_{\Lambda}-\mu_{B}\right)}{T}\right)\right]
$$

with the temperature $T$, the baryo-chemical potential $\mu_{B}$, the degeneracy factors $g_{i}$, the production volume for making the associate pair $V$ (see [21, 22]) and the total energies $E_{i}$. This formula, simplified for demonstration purpose, neglects other combinations leading to the production of $K^{+}$as well as the use of Bose-Fermi distributions, which are all included in the computation. The corresponding formula for $K^{-}$production is similar, but does not depend on $\mu_{B}$. This point will become important later on.

$$
\frac{M_{K^{-}}}{A_{\text {part }}} \sim \exp \left(-\frac{<E_{K^{-}}>}{T}\right)\left[g_{K^{+}} V \int \frac{d^{3} p}{(2 \pi)^{3}} \exp \left(-\frac{E_{K^{+}}}{T}\right)\right] .
$$

These formulae lead to a reduction of $K^{+}$and $K^{-}$yields as compared to the numbers calculated without exact strangeness conservation 21, 22]. Two extreme conditions can be seen from Eqs. (2) and (3) 21]. In the low-number limit, the additional term (due to the parameter $V$ ) leads to a linear rise of $M_{K^{+}} / A_{\text {part }}$ while $M_{\pi} / A_{\text {part }}$ remains constant. This is in remarkable agreement with the experimental observations shown in Fig. 5. For very high temperatures or very large $V$, the terms in brackets approach unity (see Ref. [21]) and the formulae coincide with the grand-canonical procedure.

A further test is the understanding of all particle yields. The measured yields (or particle ratios) can be described in this statistical concept by combinations of $T$ and $\mu_{B}$ shown as lines in Fig. 6 for central $\mathrm{Ni}+\mathrm{Ni}$ collisions at three incident energies. Besides the results for $\eta / \pi_{0}$ all measured particle ratios intersect within a small area reflecting $T$ and $\mu_{B}$ at freeze out. Surprisingly, even the measured $K^{+} / K^{-}$ratio fits into this representation and this ratio does not depend on the choice of the volume term $V$. 


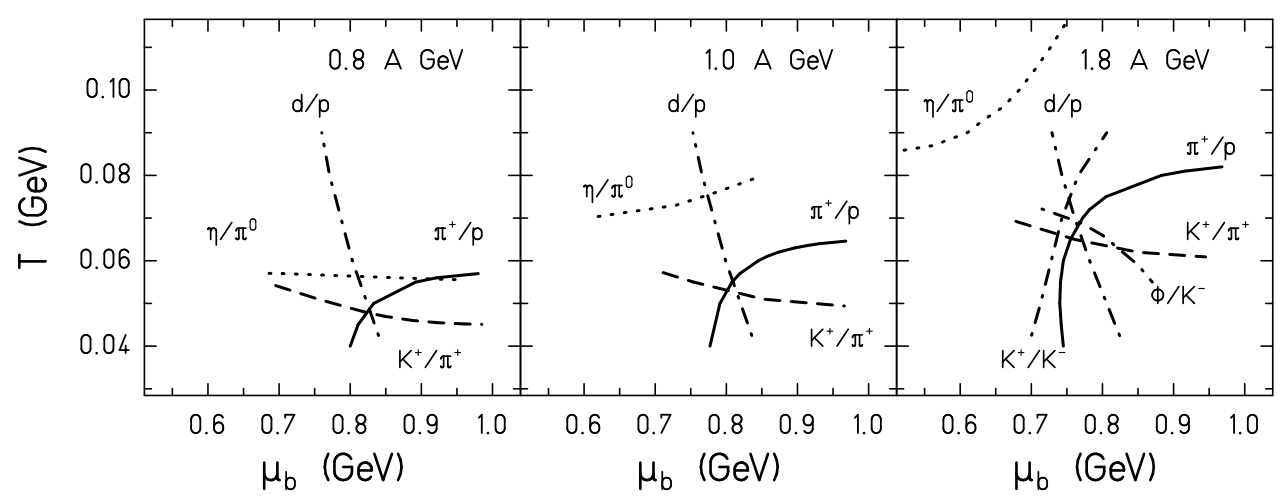

Figure 6. $T$ versus $\mu_{B}$ for central $\mathrm{Ni}+\mathrm{Ni}$ collisions from $0.8 A \cdot \mathrm{GeV}$ to $1.8 A \cdot \mathrm{GeV}$. From [21] and new, preliminary data for $K^{+}$and $K^{-} 14$.

As the $K^{+} / K^{-}$ratios are in rather good agreement with the assumption of a chemical equilibrium, it is of interest to see how the result of the thermal concept, using Ref. [29], appears in the representation as a function of $\sqrt{s}-\sqrt{s_{t h}}$ (as used in Fig. (1), which is shown in Fig. 7. Note that the x-axis extends now over a much larger range than in Fig. 1 .

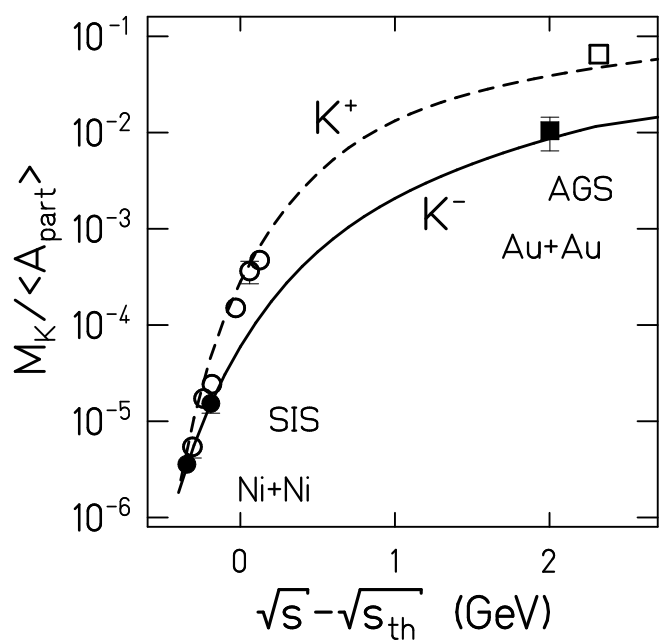

Figure 7. Calculated $K^{+} / A_{\text {part }}$ and $K^{-} / A_{\text {part }}$ ratios in the thermal model as a function of $\sqrt{s}-\sqrt{s_{t h}}$ for $\mathrm{Ni}+\mathrm{Ni}$ collisions. The points are results for $\mathrm{Ni}+\mathrm{Ni}$ collisions at SIS energies 13, 14 and $\mathrm{Au}+\mathrm{Au}$ at 10.2 $\mathrm{AGeV}$ (AGS) 24]. At AGS energies the influence of the system mass is negligible.

At values of $\sqrt{s}-\sqrt{s_{t h}}$ less than zero, the two excitation function cross. They differ at AGS energies by a factor of five. The difference in the rise of the two excitation functions can be understood by the formulae given above. The one for $K^{+}$production contains $\left(E_{\Lambda}-\mu_{B}\right)$ while the other has $E_{K^{+}}$in the exponent. As these two values are different, the excitation functions, i.e. the variation with $T$, exhibit different rises.

Furthermore, the two formulae predict that the $K^{+} / K^{-}$ratio for a given collision should not vary with centrality as $V$ cancels in the ratio. Indeed, this has been observed in $\mathrm{Au}+\mathrm{Au}$ collisions between 4 and $10.2 \mathrm{~A} \cdot \mathrm{GeV}$ [24, 25] and also at SIS energies [14]. Two examples are shown in Fig. 8. This independence of centrality is rather astonishing as one expects an influence of the different thresholds and the density variation with centrality, especially at $1.93 \mathrm{~A} \cdot \mathrm{GeV}$ where the $K^{+}$production 

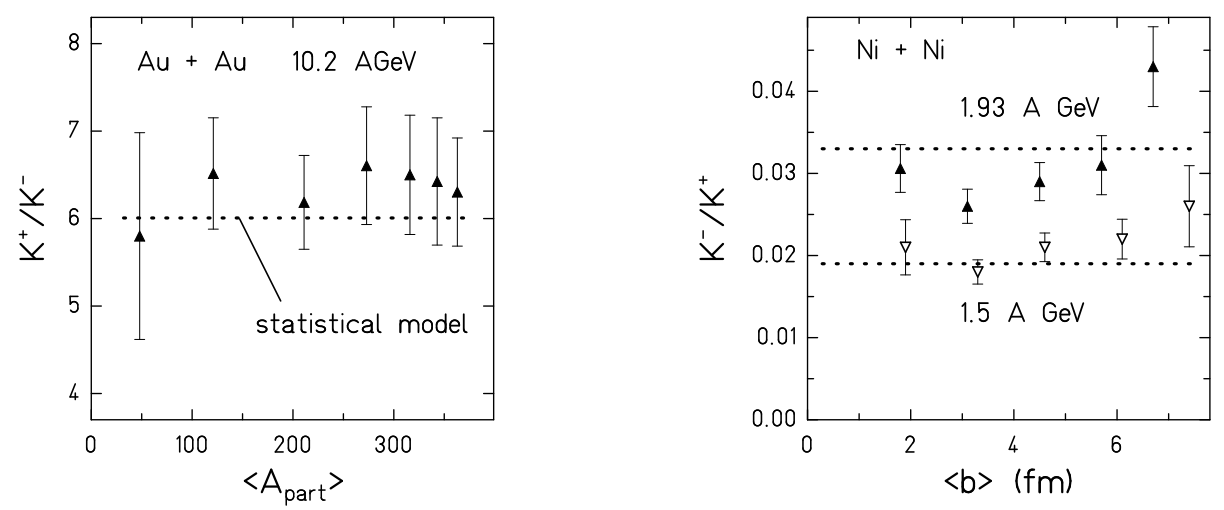

Figure 8. Ratio of $K^{+}$to $K^{-}$as a function of the number of participants $A_{p a r t}$ (left for $\mathrm{Au}+\mathrm{Au}$ at $10.2 A \cdot \mathrm{GeV}$ [26], right for $\mathrm{Ni}+\mathrm{Ni}$ at 1.5 and $1.93 A \cdot \mathrm{GeV}$ [14] together with the statistical model (dashed line) evidencing the independence of $<A_{\text {part }}>$.

is above, the $K^{-}$production below their respective thresholds.

Transport-model calculations and other estimates show clearly that strangeness equilibration requires a time interval of $40-80 \mathrm{fm} / c$ [27, 28]. While the statistical model is quite successful describing the particle yields, including strange particles, this cannot be taken as proof for chemical equlibration. Especially in case of the $K^{+}$ production, no strong absorptive channel seems to be available. This point clearly deserves further attention. At these low incident energies the strange quarks are found only in a few hadrons. The $\bar{s}$ quark is found only in $K^{+}$, while the $s$ quark will be shared between $K^{-}$and $\Lambda$ (or other hyperons). The latter sharing might be in chemical equlibrium as the reactions

$$
\pi^{0}+\Lambda \rightleftharpoons p+K^{-} \quad \text { or } \quad \pi^{-}+\Lambda \rightleftharpoons \mathrm{n}+\mathrm{K}^{-}
$$

are strong and have only slightly negative $\mathrm{Q}$-values of $-176 \mathrm{MeV}$. If these reactions are the dominating channel, the law of mass action might be applied giving for the respective concentrations

$$
\frac{[\pi] \cdot[\Lambda]}{\left[K^{-}\right] \cdot N}=\kappa .
$$

As the number of $K^{-}$relative to $\Lambda$ is small, $[\Lambda]$ can be approximated by $\left[K^{+}\right]$and rewriting gives

$$
\frac{\left[K^{-}\right]}{\left[K^{+}\right]} \propto M\left(\pi^{0}+\pi^{-}\right) / A .
$$

Figure 9 tests the validity of this relation at SIS and AGS energies showing a rather linear rise of $K^{-} / K^{+}$with $M\left(\pi^{-}+\pi^{0}\right) / A$ reflecting a constant value of $\kappa$.

\section{Beyond the Statistical Model}

While the presented results exhibit an astonishing agreement with the statistical model there are clear experimental observations which are beyond the statistical description indicating new phenomena. These results are presented by the following talks: 


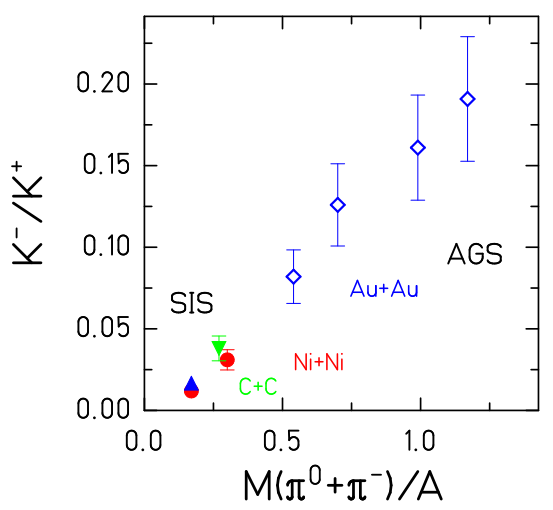

Figure 9. The $K^{-} / K^{+}$ ratio as a function of the pion multiplicity $M\left(\pi^{-}+\right.$ $\left.\pi^{0}\right) / A$ as a test of the law of mass action. Preliminary data.

- The emission of positively charged kaon, measured by the KaoS Collaboration, exhibits an elliptic flow which is hard to understand as the $K^{+}$interaction with nuclear matter is small [30]. Only by including a repulsive $K^{+} N$ potential the experimental data can be described. Talk by Y. Shin.

- The measured $p_{t}$-dependence of the directed flow of $K^{+}$in $\mathrm{Ru}+\mathrm{Ru}$ and $\mathrm{Ni}+\mathrm{Ni}$ collisions near threshold, studied by the FOPI collaboration, can only be decribed when using a repulsive $K^{+} N$ potential [31]. Talk by P. Crochet. An extremely strong antiflow signal of $K^{0}$ is found in $\mathrm{Au}+\mathrm{Au}$ collisions at $6 A \cdot \mathrm{GeV}$ by the EOS collaboration [32]. Talk by P. Chung.

Direct experimental evidence for the time evolution of pion emission is presented based on the shadowing of spectator matter in certain space-time regions [33]. In peripheral collisions of $\mathrm{Au}+\mathrm{Au}$ at $1.0 \mathrm{~A} \cdot \mathrm{GeV}$ incident energy the moving spectator matter acts like a shutter of a camera shielding the pions, i.e. modifying the pion emission pattern according to the spatial distribution of the spectator matter at the time of the pion freeze out. The motion of the spectator serves as a calibrated clock. This analysis suggests that in $\mathrm{Au}+\mathrm{Au}$ collisions at $1 A \cdot \mathrm{GeV}$ most of the high-energy pions freeze out within $10 \mathrm{fm} / c$ after the first touch of the nuclei. Low-energy pions do freeze out later and over a longer time interval [33].

\section{Summary}

The measured multiplicities of $K^{+}$and $K^{-}$production at subthreshold energies rises more than linearly with system mass $A$ and with the number of participating nucleons $A_{\text {part }}$. The spectra do not show deviations from Boltzmann shapes.

A global survey of the spectral shapes and yields of the emitted pions, $K^{+}$ and $K^{-}$points towards an interpretation within a statistical concept. This model takes into account exact strangeness conservation, i.e. the associate production of two strange particles (e.g. $K^{+}$and $\Lambda$ ). It does not only explain the various particle ratios (except $\eta$ ), but also describes the very different $A_{\text {part }}$-dependences of the $K^{+}$and $\pi$ multiplicities.

Despite the apparent success of the statistical model of particle production under the assumption of thermal and chemical equilibration and using masses of free particles, the present understanding of hadronic interactions contradicts chemical 
equilibrium for strange particles [28]. While for $K^{-}$the channel $K^{-}+N \rightleftharpoons \Lambda+\pi$ is open, for $K^{+}$no corresponding channel is available needed to understand how equilibrium can be obtained. The kaon yields in the statistical model are based on free masses, while transport model largely under-predict the measured $K^{-}$yields when using bare masses. Only if in-medium masses for $K^{-}$are used, which are considerably lower, agreement with the measured rates can be achieved. This discrepancy seem to put into question our present understanding of interactions at the high densities reached in heavy ion collisions.

Furthermore, the observation of a strong elliptic flow of $K^{+}$, and of pronounced $p_{t^{-}}$ dependence of a directed flow can only be described using a repulsive $K^{+} N$ potential.

The discrepancy between the two controversial interpretation needed to describe the data might disappear when the collision process is understood as a dynamical evolution; in some observables, e.g. particle ratios, the dynamics is lost. However, when looking at small areas of the phase space the influence of a dynamical evolution could be traced back.

[1] A. Gobbi et al., Nucl. Instrum. Methods. A 324 (1993) 156.

[2] P. Senger et al., Nucl. Instrum. Methods. A 327 (1993) 393.

[3] F. Laue, C. Sturm et al., Phys. Rev. Lett. 82 (1999) 1640.

[4] C. Sturm et al., submitted to Phys. Rev. Lett.; C. Sturm, Ph.D.Thesis, Technische Universität Darmstadt, 2000.

[5] C. Müntz et al., Z. Phys. A 357 (1997) 399.

[6] D. Pelte et al., Z. Phys. A 357 (1997) 215 ; Z. Phys. A 359 (1997) 55.

[7] H. Oeschler et al., Acta Physica Polonica B 31 (2000) 227.

[8] W. Weinhold, B. Friman, W. Nörenberg, Phys. Lett. B433 (1998) 236.

[9] B. Hong et al., Phys. Lett. B 407 (1997) 115.

[10] A. Förster, master's thesis, Technische Universität Darmstadt, 1998.

[11] C. Fuchs, A. Faessler, E., Zabrodin, Y.M. Zheng, submitted to Phys. Rev. Lett.

[12] C. Hartnack and J. Aichelin, Proceedings of the International Workshop "Hadrons in Dense Matter", Hirschegg, Austria, January 2000.

[13] R. Barth et al., Phys. Rev. Lett. 78 (1997) 4007.

[14] M. Menzel, Ph.D.Thesis, Universität Marburg, 2000.

[15] A. Förster, Ph.D.Thesis, Technische Universität Darmstadt, in preparation.

[16] R. Averbeck et al.. Z. Phys. A 359 (1997) 65; R. Averbeck et al., TAPS workshop, St. Odile, Sept. 1997 nucl-ex/9803001 and references therein.

[17] W. Cassing et al., Nucl. Phys. A614 (1997) 415.

[18] P. Braun-Munzinger, J. Stachel, J.P. Wessels and N. Xu, Phys. Lett. B 344 (1995) 43.

[19] J. Cleymans and H. Satz, Z. Phys. C57 (1993) 135.

[20] R. Hagedorn, CERN Yellow Report 71-12 (1971).

[21] J. Cleymans, H. Oeschler, K. Redlich, Phys. Rev. C59 (1999) 1663.

[22] J. Cleymans, H. Oeschler, K. Redlich, Phys. Lett. 485 (2000) 27.

[23] M. Mang, Ph.D.thesis, University of Frankfurt, 1997.

[24] L. Ahle et al., (E-802 Collaboration). Phys. Rev. C58 (1998) 3523;

[25] J.C. Dunlop and C.A. Ogilvie, nucl-th/9911015; J.C. Dunlop, Ph.D.Thesis, MIT, 1999.

[26] L. Ahle et al., (E-802 Collaboration), Phys. Rev. C60 (1999) 044904.

[27] P. Koch. B. Müller and J. Rafelski. Phvs. Rep. 142 (1986) 167.

[28] E.L. Bratkovskaya et al., nucl-th/0001008.

[29] J. Cleymans and K. Redlich, Phys. Rev. Lett. 81 (1998) 5284; Phys. Rev. C60 (1999) 054908.

[30] Y. Shin et al., Phys. Rev. Lett. 81(1998) 1576.

[31] P. Crochet et al., Phys. Lett. B486 (2000) 6.

[32] P. Chung et al., Phys. Rev. Lett. 85 (2000) 940.

[33] A. Wagner et al., Phys. Rev. Lett. 85 (2000) 18; A. Wagner, Ph.D.thesis, Technische Universität Darmstadt, 1997. 